\title{
Research On Wind Energy Resource Distribution of Yangtze Route for Dual-use Sail
}

\author{
Zhenfei Huang ${ }^{1,2, a}$, Yu Fu ${ }^{1,2, ~ b}$, Li Xu ${ }^{1,2, c}$, Bing Tang ${ }^{1,2, d}$ \\ ${ }^{1}$ School of Energy and Power Engineering of Wuhan University of Technology, \\ Wuhan, 430063, China \\ ${ }^{2}$ Reliability Engineering Institute, Wuhan University of Technology, Wuhan, 430063, China \\ ahuangzhen825725035@163.com, ${ }^{b}$ william-1232@163.com, cxuli@whut.edu.cn, \\ tangbing93@163.com
}

Keywords: Wind energy resource; Dual-use sail; Yangtze route; Rayleigh distribution model Abstract. Wind give the ship with sails auxiliary thrust, and the distribution route of wind energy resource has a significant impact on energy saving of ship with dual-sail. Rayleigh wind speed distribution model of Nanjing, Shanghai and Nantong city on the Wuhu to Shanghai route segments of Yangtze route is established in this dissertation. And on this basis, wind resource of those areas is analyzed. The results show that Wind energy resource distribution Wuhu to Shanghai leg is not balanced, and coastal areas are relatively rich, in general for sea-river-through ship with dual-use sail, the wind resources on the segment are worth exploitation.

\section{Introduction}

Wind as a driving force of the ship, in the development process of the ship has been playing an important roles, however, in the development of modern shipping, the use of wind resource has been ignored. Under the background of global warming and energy crisis, wind energy as a renewable energy re-entered the spotlight of people. How to conserve energy and improve the operation of the ship economy has become increasingly concerned about the issue of the shipping industry ${ }^{[1]}$. As technology advances, to the shipping industry, wind can be used to navigation aids, and it can be used to generate electricity to provide some power to the ship, as well. Sea-river-through ship with dual-use sail (sail can switch between navigation aids and power generation) is a new kind of green ship which can use wind energy efficiently.

Yangtze route is the "golden channel" of china and the economic exchanges artery between western of china and world ${ }^{[2]}$. And it is the main route of sea-river-through ship with dual-use sail. Wind resource distribution of this route has a major impact on sailing performance of sea- river-through ship with dual-use sail. This paper studies the wind resource distribution of Wuhu to Shanghai leg of the Yangtze route, selecting Nanjing, Nantong and Shanghai of this segment to research the wind energy resources of the three places. And this research can provide a reference for the use of wind energy of this segment.

\section{Probability distributions model of the speed of wind}

Rayleigh distribution is introduced. There are several kinds of probability distribution model of the speed of wind. For example, Weibull distribution, Rayleigh distribution, Lognormal distribution, Burr distribution, Gaussian distribution and Gamma distribution. Weibull distribution is a kind of unimodal distribution function with two parameters. The speed of wind is submitted the Weibull distribution. Its probability density function can be given as

$$
f_{w}(v)=\left(\frac{b}{c}\right)\left(\frac{v}{c}\right)^{b-1} \exp \left[-\left(\frac{v}{c}\right)^{b}\right] \quad v \geq 0 \quad b>0 \quad c>0
$$

In this function, $\mathrm{b}$ is the shape parameter, and $\mathrm{c}$ is the scale parameter. 
In general wind conditions $b=1 \sim 3$, and model is Rayleigh distribution when $b=2$. Its probability density function can be expressed as follow.

$$
f_{R}(v)=\frac{2 v}{c^{2}} \exp \left[-\left(\frac{v}{c}\right)^{2}\right]
$$

Calculation method about parameter of Rayleigh distribution is introduced. Rayleigh distribution is a special form of Weibull distribution when the shape parameter is 2 , so the estimation methods of parameter of Weibull distribution are equally applicable to the Rayleigh distribution. There are many methods to estimate the parameters of Weibull distribution. Depending on the wind conditions of statistics used different choices can be made. In this paper, the method of average wind speed is used.

The average wind speed can be calculated by equation (3).

$$
\bar{v}=\frac{1}{N} \sum_{i=1}^{N} v_{i}
$$

In the expression of Eq. (3), $v_{i}$ denotes the $i$ measurements of wind speed, $\mathrm{m} / \mathrm{s} ; N$ represents the total number of measurements.

Scale parameter c can be obtained by the following equation (4).

$$
c=\bar{v}\left[\Gamma\left(\frac{1}{b}+1\right)\right]^{-1}
$$

In this equation, $\Gamma$ is Gamma function. Gamma function $\Gamma(\beta)$ can be represented as

$$
\Gamma(\beta)=\int_{0}^{\infty} x^{\beta-1} \exp (-x) d x
$$

\section{Wind speed profile of Shanghai, Nanjing and Nantong}

According to the date of weather station of Shanghai, Nanjing and Nantong, the dates of monthly average wind speed of ten meters high of those places of 2010 are obtained. Wind speed changes with the height. The relationship between height and wind speed can be represented by Eq. (6).

$$
\frac{v_{2}}{v_{1}}=\left(\frac{H_{2}}{H_{1}}\right)^{\alpha}
$$

In this equation $\alpha$ represents the wind shear exponent, $\alpha=0.1 \sim 0.4$. In general $\alpha$ takes $0.19 ; H_{2}$ is the height corresponding to the wind speed $v_{2}, \mathrm{~m} ; H_{l}$ is the height corresponding to the wind speed $v_{1}, \mathrm{~m}$. The average height of the dual-use is about 20 meters. The monthly average wind speed of 20 meters of 2010 of Shanghai, Nanjing and Nantong are calculated, as shown in Fig.1. 


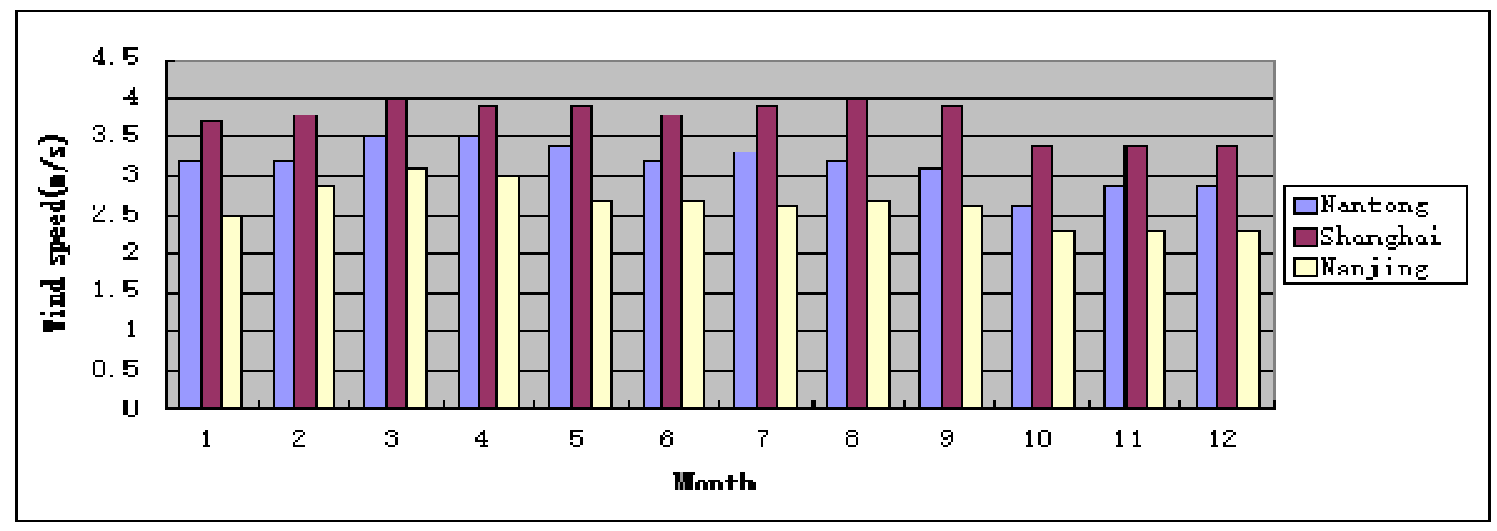

Fig.1. Monthly average wind speed

\section{Characteristics of wind energy resource of Nantong, Nanjing and Shanghai}

The parameters for evaluating wind speed are calculating. Available time of wind energy is an important indicator of the evaluation of the wind resources, which can be calculated by equation $(7)^{[3]}$.

$$
T_{w a}=\int_{v_{1}}^{v_{2}} f(v) d v=8760\left\{\exp \left[-\left(\frac{v_{1}}{c}\right)^{b}\right]-\exp \left[-\left(\frac{v_{2}}{c}\right)^{b}\right]\right\}
$$

In this equation, $T$ represents the hours of a year; $v_{1}$ and $v_{2}$ represents upper and lower limits of available wind speed. When Rayleigh distribution is used, b takes 2 . In general, $v_{1}$ and $v_{2}$ are $3 \mathrm{~m} / \mathrm{s}$ and $25 \mathrm{~m} / \mathrm{s}$.

The method of average wind speed is used to estimate annual average wind speed of Nanjing, Nantong and Shanghai, the scale parameter c of Rayleigh distribution and the available time of wind energy. The results are shown in Table 1.

Table.1.Evaluation parameters of wind speed

\begin{tabular}{cccc}
\hline Parameter & Nanjing & Shanghai & Nantong \\
\hline $\bar{v} /\left(m \cdot s^{-1}\right)$ & 2.6 & 3.8 & 3.2 \\
$c /\left(m \cdot s^{-1}\right)$ & 2.92 & 4.27 & 3.60 \\
$T_{w a} / h$ & 3048.64 & 5345.18 & 4376.27 \\
\hline
\end{tabular}

Table 1 shows that the available time of wind energy of Shanghai is maximal, while the available time that wind energy has of the three places are considerable.

Probability density distribution of wind speed is analyzed. Rayleigh distribution of wind speed is used to get the probability distribution of the three places, shown as figure 2. Fig. 2 shows that when the wind speed reached $3 \mathrm{~m} / \mathrm{s}, 2.5 \mathrm{~m} / \mathrm{s}$ and $2 \mathrm{~m} / \mathrm{s}$, the Rayleigh distribution of Shanghai, Nanjing and Nantong are maximal. Probability of the appearance of the effective wind speed which is faster than $3 \mathrm{~m} / \mathrm{s}$ of Shanghai is more than $50 \%$. The probability of Nantong is slightly less than $50 \%$. The probability of Nanjing is minimal. It is about $40 \%$. 


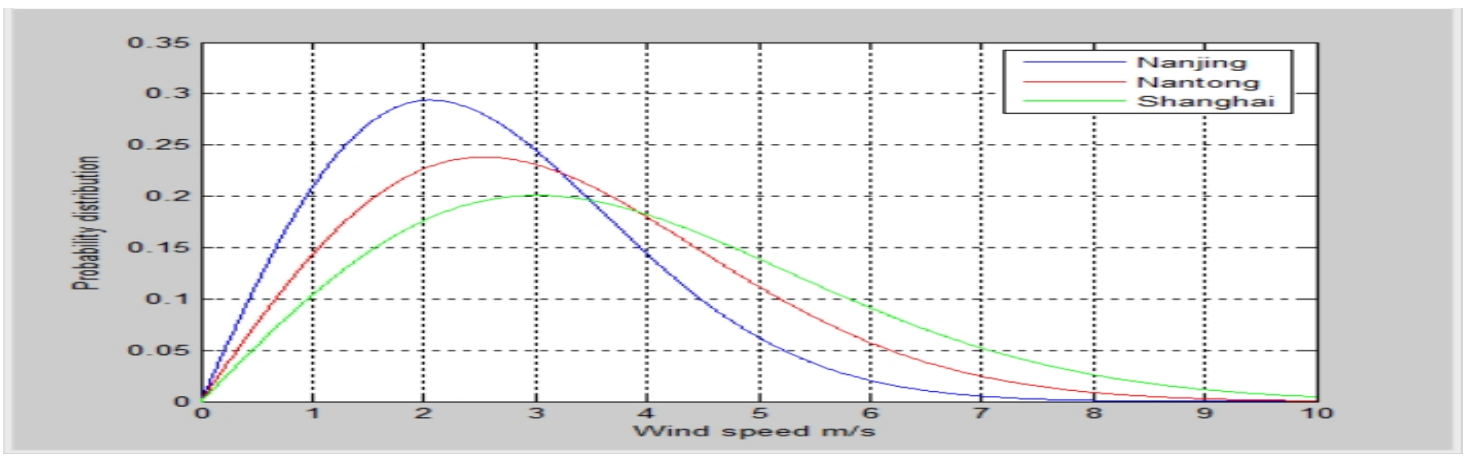

Fig.2. Probability density distribution of wind speed of Nanjing, Nantong and Shanghai

Calculate wind power density. The power obtained when vertical airflow blow on the unit cross-sectional area is called wind power density. Wind power density is a very important indicator for evaluating the wind energy resource. Wind power density can be calculated by equation (8) ${ }^{[4]}$.

$$
P(v)=\frac{1}{2} \rho v^{-3}
$$

In this equation, $P(v)$ represents wind power density, $\mathrm{W} / \mathrm{m}^{2}$. And $\rho$ is density of air. It can be calculated as follow.

$$
\rho=\frac{P}{R T}
$$

In this equation, $\mathrm{P}$ which represents the annual average atmospheric pressure take $1.0133105 \mathrm{~Pa} ; \mathrm{R}$ which is the gas constant take $287 \mathrm{~J} / \mathrm{kg}$; T that represents the annual average temperature of Shanghai, Nanjing and Nantong take 290.15K.

In practice, the wind power density depends on the probability density function. When the wind speed probability distribution submits Rayleigh distribution, the wind power density can be calculated by equation (10).

$$
P_{R}=\int_{0}^{\infty} P(v) f_{R}(v) d v=\frac{1}{2} \rho \int_{0}^{\infty} v^{3} f_{R}(v) d v=\frac{3}{\pi} \rho v^{-3}
$$

Rayleigh distribution model is used to analysis the wind power density of Shanghai, Nanjing and Nantong. The monthly average power density and annual average wind power density and are showed as figure 3 and figure 4. From March to September, wind power density of those places is larger. And wind power density of Shanghai and Nantong are relatively larger than Nanjing.

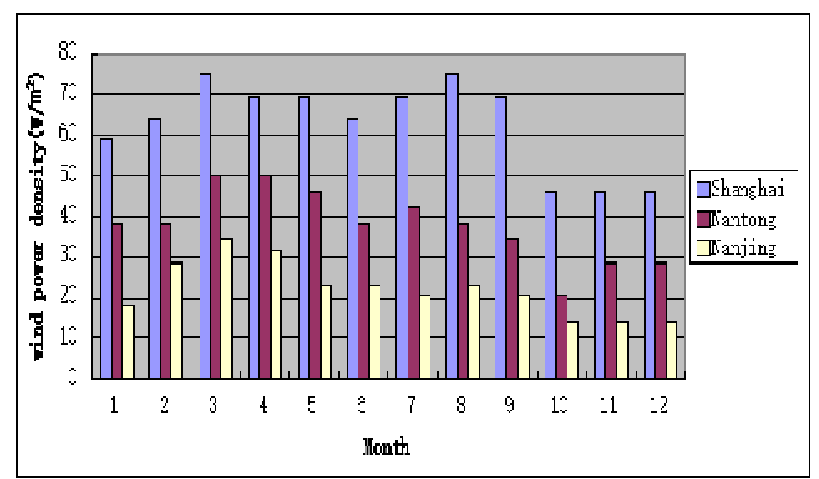

Fig.3.Monthly average wind power density

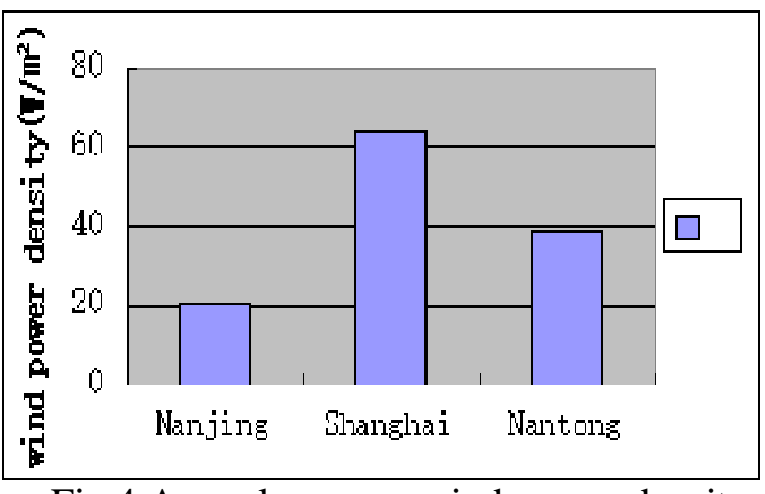

Fig.4.Annual average wind power density 


\section{Conclusion}

Rayleigh distribution model is used to evaluate the wind energy resource distribution of Shanghai, Nanjing and Nantong beside the segment which is from Wuhu to Shanghai of Yangtze route. The following conclusions can be obtained.

(1)The probability of effective wind speed which is faster than $3 \mathrm{~m} / \mathrm{s}$ of the segment that is from Wuhu to Shanghai are different in different places. The probability of coastal places is greater.

(2)On the basis of this model, the annual and monthly average wind power density is calculated. That the wind energy resource distribution of this segment is not balanced is revealed. The wind energy resource of coastal areas such as Shanghai is more abundant, and inland areas such as Nanjing are relatively smaller. And wind power density of months that are from March to September is relatively larger than other months.

(3)The available time of wind energy of Nanjing, Nantong and Shanghai are relatively considerable. Wind energy as a green energy, can be used by dual-sail to save fuel and reduce carbon dioxide emissions from ships. It has high environmental value. Overall wind energy resource on this leg is worth developing

\section{Acknowledgment}

The work was financially supported by The National Key Technology Support Program (No: 2014BAG04B01)

\section{References}

[1] Yuankui Li, Xingjun Zhang, Xingwang Yue, et al. Ocean Wind Resource Analysis Method for Wind-assist Sailing[J].Navigation of China, 2013, 36(03):90-94.

[2] Zhongzhen Yang, Xiadan Dong, Liquan Guo. Optimization of Container Liner Network on The Yangtze River[J]. Journal of Dalian Maritime University, 2014, 40(03):1-7.

[3] Yan Yan, Chang Xu, Deyou Liu, et al. Study on Probability Distribution Parameters of Wind Speed Influenced by Different Anemometer Time Intervals[J]. Renewable Energy Resources, 2011, 29(06):24-28.

[4] Mostafaeipour A, Sedaghat A, Dehghan-Niri AA, et al. Wind Energy Feasibility Study for City of Shahrbabak In Iran[J]. Renewable and Sustainable Energy Reviews, 2011, 15(6):2545-2556. 